\title{
Nail gun penetrating injury of the heart mimicking an acute coronary syndrome
}

\author{
Chiara Comoglio • Fabrizio Sansone • \\ Massimo Boffini • Marco Ribezzo • Mauro Rinaldi
}

Received: 7 June 2009 / Accepted: 29 November 2009/Published online: 5 February 2010

(C) Springer-Verlag London Ltd 2010

\begin{abstract}
We describe the case of a 75-year-old man admitted to hospital for chest pain and syncope. Physical examination was normal with evidence of a very small wound on the left chest. Considering the presence of multiple coronary risk factors, an acute coronary syndrome was initially suspected, but the electrocardiogram (EKG) was normal and only a slight increase of cardiac enzymes was detected. The hypothesis of aortic dissection was also considered and in order to discriminate between the aortic and coronary syndrome, a thoracic and coronary computed tomography (CT) scan was performed. The CT scan showed a metallic structure, suggestive of a nail, about $6 \mathrm{~cm}$ in length, in the deep layers of the left ventricular wall and a small pneumothorax due to a lung lesion. The patient was therefore transferred to our department for urgent cardiac surgery that was performed without complications.
\end{abstract}

Keywords Chest trauma - Nail gun penetrating injury . Trauma management . Acute coronary syndrome .

Aortic dissection

\section{Introduction}

Accidents with nail guns usually cause orthopaedic lesions of the non-dominant arm, but just a few cases of cardiac

C. Comoglio $\cdot$ F. Sansone $(\triangle) \cdot$ M. Boffini $\cdot$ M. Ribezzo $\cdot$

M. Rinaldi

Division of Cardiac Surgery, University of Turin,

San Giovanni Battista Hospital,

C.so Bramante 88 ,

10127 Turin, Italy

e-mail: fabrisans@katamail.com injuries have been reported. The incidence of self-inflicted cases is actually rising and some cases have been reported. The right ventricle is most frequently involved because of its anterior location [1], but the aorta and other mediastinal structures may be damaged. Although these injuries are now considered survivable, the mortality rate of nail gun injuries reported in the literature is about $25 \%$ [2].

\section{Clinical summary}

We describe the case of a 75-year-old man with multiple cardiac risk factors like hypertension, diabetes mellitus, and smoking who experienced an episode of chest pain and syncope while he was working on some domestic jobs. He was hospitalized for suspected acute coronary syndrome (ACS). Physical examination was normal except for the presence of a small wound on the left chest wall whose origin the patient could not explain. The electrocardiogram (EKG) was normal, and cardiac enzymes were slightly increased (troponin T $0.12 \mathrm{ng} / \mathrm{ml}$ ). To discriminate between ACS and aortic syndrome, thoracic and coronary computed tomography (CT) scanning was performed. CT scanning revealed a nail of about $6 \mathrm{~cm}$ in length in the deep layers of the left ventricular wall and severe calcification of the left anterior descending artery (LAD) without coronary lesions due to the nail (Fig. 1a, b). Echocardiography showed mild pericardial effusion without signs of cardiac tamponade, normal ejection fraction (EF), and the presence of a foreign body in the deep layers of the lateral wall. The patient was transferred to our department for urgent cardiac surgery. He was stable and in order to clarify the LAD calcifications, coronary angiography was performed (Fig. 1b). It showed normal coronary arteries and confirmed the presence of a nail in the lateral wall. The patient underwent surgery: we 
Fig. 1 a CT scan. b Coronarography showed the nail in the lateral wall
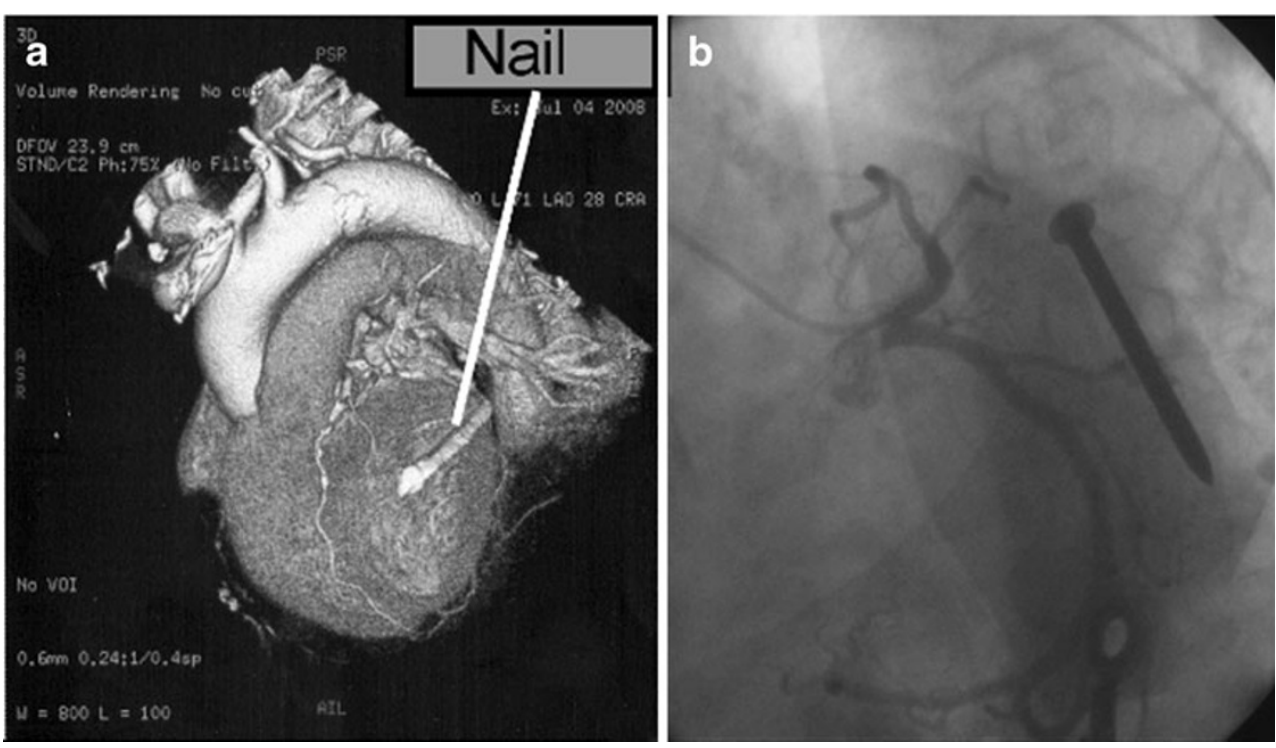

found the nail in the deep layers of the left ventricular wall with the tip protruding from the posterolateral wall (Fig. 2a, b). Moreover, a lesion of the left lung was found. The nail was gently removed and the lesions of the heart, at the entry and exit points, were repaired with a Prolene $4 / 0$ stitch on a Teflon felt. A TachoSil sponge was applied in order to optimize hemostasis. Thepulmonary lesion was also treated with a TachoSil sponge to control the air leak.

Questioning the patient about the accident, he remembered only to have been working with a nail gun. The hypothesis of an accidental injury appeared to be the most likely, but during the postoperative period, many psychological evaluations supported the hypothesis of an attemp- ted suicide due to latent depression. The postoperative course was uneventful: the patient was discharged on the 10 th postoperative day without complications.

\section{Discussion}

Nail gun injuries are common as a trauma of the arms or legs, but very few reports of heart injury are described. Given the remote settings in which these devices are often used, attention to the prevention of nail gun injuries should increase. Most of the nail guns are provided with security systems; nevertheless, accidental injury may occur [3].
Fig. 2 a Location of the nail penetrating into the wall of the left ventricle. b The nail after removal
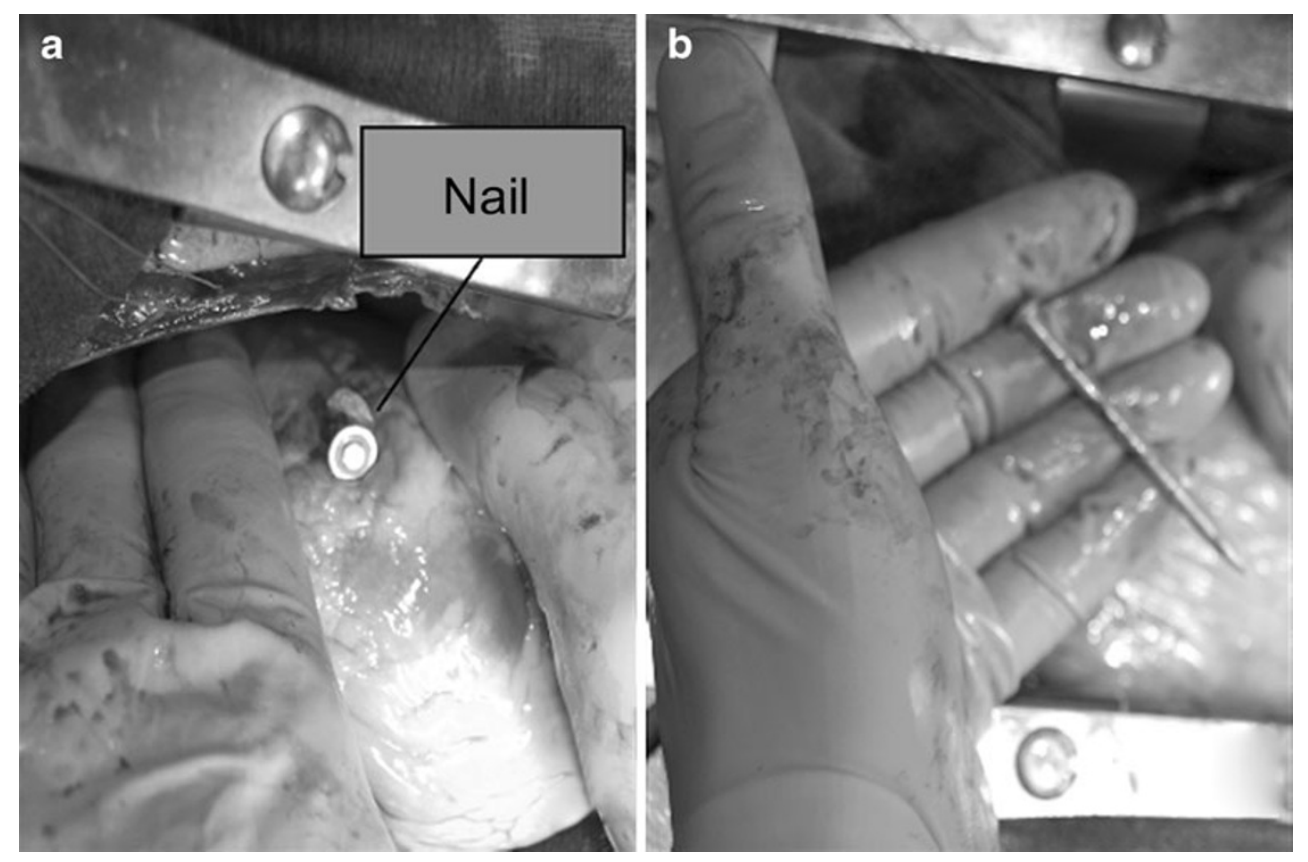
As in our case, the patient was initially referred to a peripheral hospital without a cardiac surgery division and at the moment of the diagnosis, many problems concerning patient management emerged. However, the patient was clinically stable and the transfer to our cardiac surgery division was uneventful.

In our case, the left ventricular involvement was the most unusual finding, very rarely reported in the literature, maybe because the patient pushed the nail shot from the gun into the left chest wall laterally. Moreover, the left ventricular lesion did not bleed and the patient did not suffer from cardiac tamponade, as usually happens in cases like this.

\section{Conclusion}

In conclusion, nail gun injuries to the heart, even though rare, can occur and are survivable. Despite being injuries caused by a projectile, mortality and treatment appear similar to those of low-velocity mechanisms of injury, such as stab wounds. This case demonstrates that physical examination plays a fundamental role and also the examination of chest wall may give in some cases useful elements for diagnosis. Even though a chest X-ray should offer some information about nail presence, CT scanning is mandatory for the evaluation of heart damage and nail location. Our experience with the present case demonstrates that left ventricular involvement may be possible and the key to survival is expedient care that recognizes the physical nature of the injury.

\section{References}

1. Eren E, Keles C, Sareyyupoglu B, Bozbuga N, Balkanay M, Yakut C (2004) Penetrating injury of the heart by a nail gun. J Thorac Cardiovasc Surg 127:598

2. Asensio JA, Berne JD, Demetriades D, Chan L, Murray J, Falabella A et al (1998) One hundred five penetrating cardiac injuries: a 2year prospective evaluation. J Trauma 44:1073-1082

3. Karger B, Teige K (1995) Suicide with a nail gun device: wound ballistics and wound entry morphology (in German). Arch Kriminol 195:153 